Trauma Berufskrankh 2006 · 8 [Suppl 1]: S58-S64 DOI 10.1007/s10039-006-1102-6

Online publiziert: 28. Januar 2006

๑) Springer Medizin Verlag 2006
J. Sturm ${ }^{1}$ - C. A. Kühne ${ }^{2}$. S. Ruchholtz ${ }^{2}$

${ }^{1}$ Unfallchirurgie, Klinikum Lippe-Detmold

${ }^{2}$ Klinik für Unfallchirurgie, Universitätsklinikum, Essen

Mitglieder der "Initiative Traumnetzwerk": s. Fußnote

\title{
Initiative Traumanetzwerk
}

\section{Qualitätssichernde Maßnahmen bei der Polytraumaversorgung}

\section{Inzidenz schwerer Verletzungen in Deutschland}

In der Bundesrepublik Deutschland ereignen sich jährlich 7-8 Mio. Unfälle [19]. Nach aktuellen Berechnungen erleiden davon jährlich etwa 33.000-38.00o Patienten ein Polytrauma (ISS $\geq 16$ ) [19, 27, 31]. Da$\mathrm{zu}$ ist eine vergleichbar hohe $\mathrm{Zahl}$ an $\mathrm{Pa}$ tienten anzunehmen, die zwar leichter verletzt sind (ISS $<16$ ), jedoch initial aufgrund des Unfallmechanismus oder einer vorübergehenden Störung der Vitalparameter über den Schockraum einer Klinik aufgenommen und behandelt werden müssen.

Mit 8,5\% stehen Verkehrsunfälle zwar bezüglich der Häufigkeit an 3. Stelle, in Bezug auf die Verletzungsschwere sind sie jedoch führend [18]. Insgesamt wurden im Jahr 2003 463.170 Personen im Straßenverkehr verletzt, 6613 hiervon tödlich [51]. Durchschnittlich verstarben damit $1,4 \%$ aller Unfallverletzten.

\section{Versorgungsqualität schwer verletzter Patienten}

Die Qualität der Polytraumaversorgung in Deutschland ist - bedingt durch geografische und infrastrukturelle Unterschiede zwischen den Bundesländern und durch die Varianz der Behandlungskonzepte in einzelnen Krankenhäusern - durchaus sehr inhomogen. Berechnet für die einzelnen Bundesländer verstarben in Deutschland zwischen $0,4 \%$ (Hamburg) und 2,7\% (Mecklenburg-Vorpommern) aller im Straßenverkehr verunfallten Patienten. Die neuen
Bundesländer lagen hier meist deutlich über dem bundesweiten Durchschnitt [27].

Neben den Problemen der Verteilung der Krankenhäuser und den Unterschieden in den präklinischen Rettungswegen ist entsprechend der Daten aus dem Traumaregister der Deutschen Gesellschaft für Unfallchirurgie (DGU) die Qualität der Behandlung schwerverletzter Patienten in verschiedenen lokalen Versorgungssystemen mitunter von deutlicher Varianz [42].

Analysen aus den Vereinigten Staaten zur Qualität der klinischen Versorgung schwerverletzter Patienten konnten zeigen, dass durch die Einführung „regionalisierter“ Traumasysteme die Rate vermeidbarer Todesfälle bei der Behandlung schwer verletzter Patienten um 50\% reduziert werden konnte [6, 7, 36, 48, 49, 57]. Dabei konnte die Letalität nach schwerem Trauma um $15-20 \%$ verringert werden [ 23 , $24,37,46,50,58]$.

Aus Deutschland gibt es diesbezüglich bisher wenige vergleichbare Analysen. Biewener et al. [4] führten in der Region um Dresden eine Analyse zur Qualität der Schwerverletztenversorgung in Abhängigkeit von der Versorgungsstufe des definitiven Zielkrankenhauses durch. Hierbei zeigte sich ein signifikanter Anstieg der Letalität nach schwerem Trauma (bei gleicher Verletzungsschwere) für die Patienten, die ausschließlich in einem Krankenhaus der Grund- und Regelversorgung behandelt wurden.

Aber auch in universitären Traumazentren lassen sich in Deutschland Va- rianzen in der Behandlungsqualität mit Einfluss auf die Letalität nach schwerem Trauma nachweisen. Dies konnte sowohl im Rahmen interner Qualitätsmanagementsysteme als auch auf Basis einer externen Qualitätskontrolle nachgewiesen werden $[42,44]$.

\section{Strukturelle Voraussetzungen für die Polytraumaversorgung}

Die Prognose eines schwer verletzten Patienten hängt entscheidend von der möglichst zeitnahen adäquaten und prioritätengerechten Versorgung seiner Verletzungen ab. Diesbezüglich ist der möglichst schnelle Transport in ein Krankenhaus mit entsprechender Expertise von überragender Bedeutung.

Im Folgenden werden die Ergebnisse einer systematischen Analyse zur Verteilung und zur Erreichbarkeit dargestellt [27].

\section{Krankenhäuser für die Schwerverletztenversorgung}

Nach Erhebungen der Initiative Traumanetzwerkder DGU beteiligen sich derzeit bundesweit etwa 118 Krankenhäu-

Mitglieder der Initiative Traumanetzwerk sind: PD Dr. H.J. Bail (Berlin), PD Dr. A. Beck (Ulm), Prof. Dr. B. Bouillon (Köln), Dr. S. Hentsch (Koblenz), Dr. C.A. Kühne (Essen), Prof. Dr. C. Lackner (München), Prof. Dr. U. Obertacke (Mannheim), Prof. Dr. S. Ruchholtz (Essen), Prof. Dr. A. Seekamp (Kiel), Prof. Dr. J. Sturm (Detmold), Prof. Dr. C. Waydhas (Essen), Prof. Dr. A. Wentzensen (Ludwigshafen) 
ser der Maximal-, 219 Krankenhäuser der Schwerpunkt- und 439 Krankenhäuser der Grund- und Regelversorgung an der klinischen Behandlung polytraumatisierter Patienten (• Abb. 1) [27].

Durchschnittlich existieren pro Bundesland etwa 20 Maximal- und Schwerpunktkrankenhäuser zur Versorgung Polytraumatisierter. Tatsächlich allerdings differiert die Anzahl zwischen den einzelnen Bundesländern stark. So werden z. B. im Saarland nur 4 Krankenhäuser zur Polytraumaversorgung vorgehalten, entsprechend ihrer Einwohnerzahl in den 4 einwohnerstärksten Bundesländern (Nordrhein-Westfalen, Bayern, Baden-Württemberg, Niedersachsen) hingegen zwischen 19 und 63 Krankenhäuser zur (Akut)Behandlung Polytraumatisierter.

Das beste Verhältnis von Krankenhäusern pro Einwohner hat Bremen (165.00o Einwohner/Krankenhaus), das schlechteste findet sich in Brandenburg (515.00o Einwohner/Krankenhaus) [27] (s. auch Statistisches Bundesamt, http:// www.destatis.de).

\section{Versorgungsfläche pro Krankenhaus}

Umgerechnet auf das Versorgungsgebiet liegt der durchschnittliche Einzugsbereich eines Krankenhauses bei $1162 \mathrm{~km}^{2}$. Den kleinsten Versorgungsbereich haben die Stadtstaaten Berlin, Bremen und Hamburg. Von den größeren Bundesländern weist Nordrhein-Westfalen die kleinsten Einzugsgebiete - also günstigsten geografischen Versorgungsprofile - auf, die größten Einzugsgebiete - ungünstigsten geografischen Versorgungsstrukturen finden sich in den neuen Bundesländern (Brandenburg, Mecklenburg-Vorpommern) $[27]$.

\section{Straßennetz in Deutschland}

Auf $1 \mathrm{~km}^{2}$ Fläche entfallen in der Bundesrepublik durchschnittlich $1,5 \mathrm{~km}$ überörtliche Straße (Bundes- und Landstraße). Neben den Stadtstaaten Berlin, Bremen und Hamburg haben hierbei die neuen Bundesländer das schlechteste Verhältnis zwischen Fläche $\left(\mathrm{km}^{2}\right)$ und Straßennetz. Das beste Verhältnis findet sich in Nordrhein-Westfalen und RheinlandPfalz [27].

Trauma Berufskrankh 2006 • 8 [Suppl 1]: S58-S64 DOI 10.1007/s10039-006-1102-6

(c) Springer Medizin Verlag 2006

J. Sturm · C. A. Kühne · S. Ruchholtz

Initiative Traumanetzwerk. Qualitätssichernde

Maßnahmen bei der Polytraumaversorgung

\section{Zusammenfassung}

Die Qualität der Polytraumaversorgung in Deutschland ist sehr inhomogen. Derzeit sind etwa 118 Krankenhäuser der Maximal, 219 Krankenhäuser der Schwerpunkt- und 439 Krankenhäuser der Grund- und Regelversorgung in die Schwerverletztenbetreuung eingebunden. Numerisch sind somit zwar ausreichend Krankenhäuser zur Polytraumaversorgung vorhanden, allerdings bestehen erhebliche regionale Unterschiede sowohl in der Vorhaltung ausreichend ausgestatteter Traumazentren als auch in den infrastrukturellen Gegebenheiten. Um eine optimale Polytraumaversorgung in Deutschland zu gewährleisten, sollten die Versorgungsstruktur an regionale Bedürfnissen angepasst und die strukturellen und personellen Voraussetzungen in der Versorgung schwerverletzter Patienten weitestgehend standardisiert werden. Die Initiative Traumanetzwerk der DGU

\section{Initiative for a trauma network. Quality assurance in treatment of multiple injury}

\section{Abstract}

The quality of multiple trauma care in Germany varies widely, both from area to area and as a result of different treatment concepts in individual hospitals. There is a total of $776 \mathrm{in}$ stitutions providing trauma care: 118 level I, 219 level II, and 439 level III-IV trauma centres. In theory, these are enough to provide adequate multiple trauma care for the whole of the German population. At the same time, access to level I and II trauma care also differs widely in different regions in Germany. For the quality of multiple trauma care it is necessary both to tailor access to such care to needs within each region and to standardise trauma care in terms of structural and staff resources. The German Society for Trauma Surgery's initiative for a trauma network is intended to make it possible to improve and hat zum Ziel, die Qualität der Polytraumaversorgung durch die Empfehlung von strukturellen und organisatorischen Voraussetzungen (S3-Leitlinie der DGU), speziellen Ausbildungsprogrammen für Ärzte (ATLS) und Qualitätsmanagementprogrammen (Traumaregister der DGU) deutschlandweit auf ein einheitliches und flächendeckend hohes $\mathrm{Ni}$ veau zu bringen. Durch die Initiierung von lokalen Traumanetzwerkstrukturen zwischen überregionalen und regionalen Traumazentren soll sichergestellt werden, dass jeder polytraumatisierte Patient innerhalb von etwa 30 min vom Unfallort in den Schockraum eines geeigneten bzw. zertifizierten Krankenhauses transportiert werden kann.

\section{Schlüsselwörter}

Polytrauma · Versorgungsqualität · Traumazentrum · Traumaregister · Traumanetzwerk

standardise the level of trauma care in Germany, as it is hoped it will yield recommendations on structure and organisation (Guideline S3 of the German Society for Trauma Surgery), on specialised training for trauma surgeons (Advanced Trauma Life Support ${ }^{\circ}$ ) and on quality management programmes (Trauma Registry of the German Society for the Trauma Surgery). Implementation of a regional network of local trauma hospitals should mean that severely injured patients can be taken from the scene to the emergency department of a specialised and certified trauma centre within 30 minutes.

\section{Keywords}

Multiple trauma · Quality of care · Trauma centre · Trauma registry · Trauma network 


\section{Aktuelle Themen}
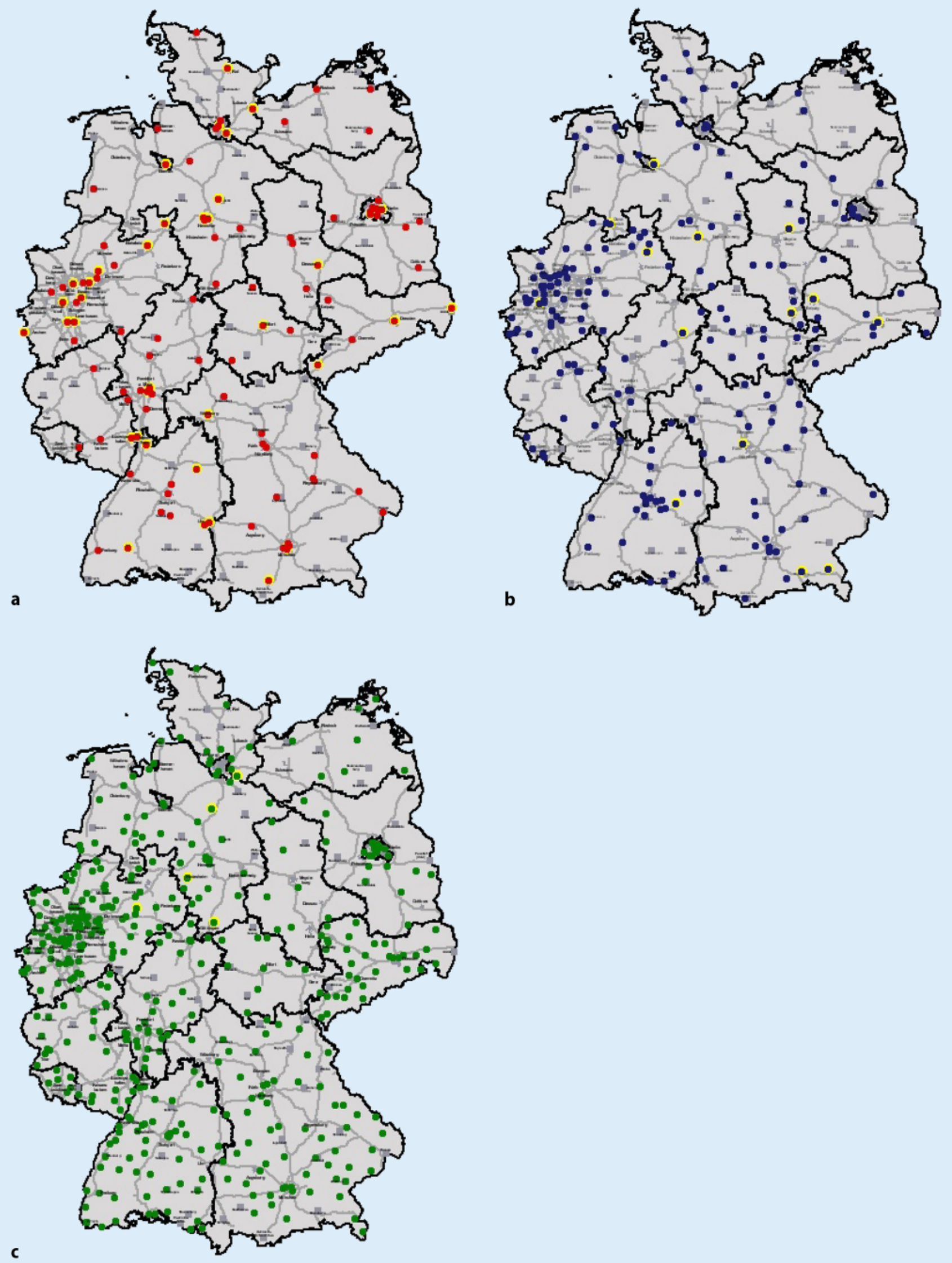

Abb. 1 A Kliniken der a Maximal-, b Schwerpunkt-, c Regelversorgung (Stand: November 2005), gelber Rand Kliniken mit Teilnahme am Traumaregister der DGU 


\section{Initiative Traumanetzwerk der DGU}

Sie hat das Ziel, die Qualität der Polytraumaversorgung durch Initiierung lokaler Traumanetzwerke flächendeckend deutschlandweit auf ein hohes Niveau zu bringen. Die Patientenallokation basiert dabei auf einem 3-stufigen Versorgungsschema von überregionalem Traumazentrum, regionalem Traumazentrum und Krankenhaus der unfallchirurgischen Grundversorgung.

\section{Aufnahmekriterien über einen Schockraum}

Um möglichst keine Zeit bis zur definitiven klinischen Versorgung zu verlieren, sollte bereits durch den Notarzt die Notwendigkeit der Behandlung in einem überregionalen bzw. regionalen Traumazentrum eingeschätzt werden können. Neben dieser klinischen Beurteilung sollten am Unfallort erhebbare Kriterien bei der Einschätzung herangezogen werden (• Tab. 1) $[8,14,15,26,47,53]$.

\section{Zielklinik polytraumatisierter Patienten}

Die aufnehmende Klinik sollte im Idealfall ein überregionales Traumazentrum sein, das die unten genannten Voraussetzungen erfüllt.

Bei hämorrhagischem Schock oder einer zu erwartenden Transportzeit von mehr als 30 min zwischen Unfallort und überregionalem Traumazentrum sollte das nächstgelegene regionale Traumazentrum angefahren werden, das entsprechend ausgestattet und zertifiziert ist.

\section{Anforderungen an die frühe klinische Polytraumaversorgung}

Schockraum. Er stellt den Ort der primären Aufnahme von potenziell schwer verletzten Patienten dar. Pro Behandlungseinheit wird eine Mindestgröße von 25-50 $\mathrm{m}^{2}$ empfohlen $[26,58]$. Die Voraussetzungen zur zeitgleichen Versorgung von 2 Schwerverletzten sollten beim überregionalen Traumazentrum gegeben sein, was somit $\mathrm{zu}$ einer $\mathrm{zu}$ fordernden Mindestgröße von $50 \mathrm{~m}^{2}$ führt [25]

Tab. 1 Kriterien am Unfallort für einen hohen Gefährdungsgrad

\begin{tabular}{|ll} 
1.Vitalwerte & Glasgow-coma-Score $<14$ (bei Traumaanamnese) \\
\hline Systolischer Blutdruck $<90 \mathrm{mmHg}$ \\
\hline Atemfrequenz $<10$ oder $>29 / \mathrm{min}$ \\
\hline Sauerstoffsättigung $<90 \%$ \\
\hline Instabiler Thorax \\
\hline Offene Thoraxverletzung \\
\hline Instabile Beckenfraktur \\
\hline$>1$ Fraktur großer Röhrenknochen der unteren Extremitäten \\
\hline Proximale Amputation \\
\hline Fußgänger oder Fahrradfahrer angefahren $(>30 \mathrm{~km} / \mathrm{h})$ \\
\hline Motorrad- oder Autounfall mit hoher Geschwindigkeit \\
\hline Ejektion aus dem Fahrzeug \\
\hline Karosserieverformung über $50 \mathrm{~cm}$ \\
\hline Tod eines Insassen \\
\hline Sturz aus mehr als $3 \mathrm{~m}$ Höhe \\
\hline Explosionsverletzung \\
\hline Einklemmung/Verschüttung \\
\hline
\end{tabular}

Tab. 2 Obligatorische Einrichtungen eines Traumazentrums

\begin{tabular}{ll} 
Abteilung & Ausstattung \\
Radiologie & Konventionelle Röntgendiagnostik \\
\cline { 2 - 2 } & Bildverstärker \\
\cline { 2 - 2 } & Angiographieeinheit \\
\cline { 2 - 2 } & Multislice-CT \\
\cline { 2 - 2 } & MRT \\
\hline Unfallchirurgie/Chirurgie/Anästhesie & $\begin{array}{l}\text { Intensivstation (Erfüllung der Voraussetzungen zur Abrech- } \\
\text { nung der Komplexbehandlung Intensivmedizin) }\end{array}$ \\
\hline Transfusionsmedizin & Neuromonitoring \\
\hline Labormedizin & Blutbank \\
\hline aBei neurochirurgischer Versorgung & Zentrallabor \\
\hline
\end{tabular}

In unmittelbarer Nachbarschaft zum Schockraum sollte sich ferner ein Raum mit der Möglichkeit zur Durchführung von Notfalleingriffen (separates Narkosegerät, Operationssiebe für unfall-, allgemein-, neuro- und thoraxchirurgische Noteingriffe) befinden.

Behandlungsteam. Das Schockraumteam sollte initial aus mindestens 3 Ärzten (Chirurg in unfallchirurgischer Weiterbildung bzw. Unfallchirurg, Assistenzarzt in chirurgischer Weiterbildung, Anästhesist) und 3 Mitarbeitern (unfall/chirurgisches, anästhesiologisches Pflegepersonal, MTRA) bestehen [12, 25, 33, 45]. Bei primärer bildgebender Diagnostik durch ein im Schockraum installiertes CT sollte zu Beginn der Versorgung auch ein Radiologe anwesend sein [9, 54]. Die Präsenz eines Oberarztes der
Unfallchirurgie sollte kurzfristig (innerhalb von $20 \mathrm{~min}$ ) gewährleistet sein [10, $11,17,22]$.

In einem überregionalem Traumazentrum muss dieses Team je nach vorliegendem Verletzungsmuster durch Vertreter anderer Fachdisziplinen erweitert werden können. Neurochirurg, Viszeralchirurg, Radiologe, Herz-Thorax-Chirurg, Mund-Kiefer-Gesichts-Chirurg bzw. HNO-Arzt, Urologe, Augenarzt und Pädiater sollten innerhalb von 20 min präsent sein können [1, 20, 25, 28, 29, 34, 35, 41, 55, 56]. Falls eine der genannten Disziplinen nicht im Traumazentrum angesiedelt ist, sollte die zeitgerechte (innerhalb von $20 \mathrm{~min}$ ) und $24 \mathrm{~h}$ durchgehende Verfügbarkeit eines Facharztes durch Kooperationen mit nahe gelegenen Kliniken sichergestellt sein. 
Tab. 3 Mögliche Kriterien für eine Weiterverlegung Schwerverletzter

\begin{tabular}{|c|c|}
\hline \multirow[t]{6}{*}{ ZNS } & Schädel-Hirn-Trauma \\
\hline & Penetrierende oder Impressionsfraktur \\
\hline & Offenes SHT \\
\hline & $\mathrm{GCS}<15$ (traumabedingt) \\
\hline & Neurologische Herdsymptomatik \\
\hline & Trauma des Rückenmarks oder größere Wirbelfraktur \\
\hline \multirow[t]{4}{*}{ Thorax } & Weites Mediastinum oder Verdacht auf Verletzung großer Gefäße \\
\hline & Schwere Verletzung der Brustwand oder Lungenkontusion \\
\hline & Kardiale Verletzung \\
\hline & Notwendigkeit längerer Beatmung \\
\hline \multirow[t]{5}{*}{ Becken/Abdomen } & Instabile Beckenringfraktur \\
\hline & Beckenringfraktur und hämorrhagischer Schock \\
\hline & Offenes Beckentrauma \\
\hline & Verletzung parenchymatöser Organe \\
\hline & Komplexe Verletzung urogenitaler Organe \\
\hline \multirow[t]{5}{*}{ Extremitäten } & Schwere offene Frakturen \\
\hline & Traumatische Amputation mit Potenzial der Replantation \\
\hline & Komplexe Gelenkfrakturen \\
\hline & Schwere Quetschverletzungen \\
\hline & Ischämie \\
\hline \multirow[t]{4}{*}{ Multiorganverletzung } & SHT mit Gesicht, Thorax, Abdomen, Becken \\
\hline & Verletzung von mehr als 2 Körperregionen \\
\hline & Schwere Verbrennung (bei > 20\% in Spezialklinik) \\
\hline & Mehrere Frakturen von großen Röhrenknochen \\
\hline \multirow[t]{5}{*}{ Komorbidität } & Alter $<5$ oder $>55$ Jahre \\
\hline & Kardiale oder respiratorische Vorerkrankung \\
\hline & Insulinpflichtiger Diabetes mellitus \\
\hline & Schwangerschaft \\
\hline & Immunsuppression \\
\hline \multirow[t]{4}{*}{ Sekundäre Verschlechterung } & Maschinelle Beatmung notwendig \\
\hline & Sepsis \\
\hline & Einfaches oder multiples Organversagen \\
\hline & Schwere Weichteilnekrose \\
\hline
\end{tabular}

Die neurotraumatologische Versorgung kann auch durch einen entsprechend ausgebildeten Chirurgen/Unfallchirurgen erfolgen $[3,16,21]$. Voraussetzung sollte jedoch die enge Kooperation mit einer Neurochirurgischen Klinik sein [39].

Ferner sollten dem erweiterten Team ein Oberarzt der Anästhesiologie sowie das Operationspersonal angehören [26].

\section{Organisatorische Voraussetzungen der} Schockraumversorgung. Die Behandlung von Schockraumpatienten sollte auf klinikinternen, interdisziplinären Behandlungsleitlinien basieren [13, 32, 38]. Die Grundlage der frühen klinischen Polytraumaversorgung stellt die S3-Leitlinie der Deutschen Gesellschaft für Unfallchirurgie dar.
Zur Ausbildung der initial im Schockraum anwesenden (Unfall-)Chirurgen empfiehlt die Deutsche Gesellschaft für Unfallchirurgie die erfolgreiche Absolvierung eines ATLS(,advanced trauma life support")-Kurses. Dessen Konzept bietet einen übergeordneten Behandlungsleitfaden, der sich an den physiologischen Bedürfnissen des Patienten orientiert [5, 52].

Neben einem übergeordneten Behandlungskonzept sollten klare, der jeweiligen Struktur des Hauses angepasste Absprachen in Form von schriftlichen Behandlungsanweisungen bestehen, die die interdisziplinäre Kooperation in der initialen Behandlungsphase regeln. Durch Implementierung von interdisziplinären Qualitätsmanagementstrukturen sollte die Schwerverletztenversorgung in Bezug auf die Effektivität der Behandlung kontinuierlich verbessert werden [45].

Weitere obligatorische Einrichtungen. In Anlehnung an die Empfehlungen zur Struktur, Organisation und Ausstattung der klinischen Patientenversorgung in unfallchirurgischen Abteilungen in Krankenhäusern der Bundesrepublik Deutschland von Haas et al. [19] aus dem Jahr 1997 sollten die in 0 Tab. 2 beschriebenen Einrichtungen in 24-h-Bereitschaft vorgehalten werden.

\section{Interaktion vernetzter Kliniken im lokalen Traumanetzwerk}

Die Initiierung und die Aufrechterhaltung lokaler Netzwerkstrukturen sollten von einem überregionalen Traumazentrum ausgehen.

Jedes regionale Traumazentrum muss mit einem überregionalen Traumazentrum in einer lokalen Netzwerkstruktur verbunden sein. In Abhängigkeit von regionalen Gegebenheiten (z. B. Krankenhausdichte) sind Kooperationen mit mehreren überregionalen Traumazentren möglich.

\section{Qualitätsmanagement im lokalen Traumanetzwerk}

Die Qualität der Polytraumaversorgung sollte durch externe Qualitätssicherungsprogramme kontinuierlich und ununterbrochen dokumentiert werden [19]. Diesbezüglich ermöglicht das Traumaregister der DGU die Beobachtung des Behandlungsprozesses bzw. -ergebnisses über mehrere Jahre [2, 43].

Durch regelmäßige Treffen eines Qualitätszirkels muss die Güte der Polytraumaversorgung in einem lokalen Netzwerk überprüft werden. Der Zirkel setzt sich aus Entscheidungsträgern bzw. verantwortlichen Ärzten und medizinischem Personal der beteiligten Kliniken zusammen. Als Grundlage der Beurteilung der Polytraumaversorgung dienen die im Traumaregister erfassten Daten.

\section{Kommunikation im lokalen Traumanetzwerk}

Telefon. Um in der akuten Behandlungssituation keine Zeit durch verzöger- 
te telefonische Verbindungen zu verlieren, sollten die Kommunikationswege zwischen den Entscheidungsträgern (Chefärzte, Oberärzte, Dienstärzte) der Kliniken eines lokalen Netzwerks durch vorhergehende Absprachen (z. B. Notfalltelefon) definiert sein.

Telemedizin. Insbesondere zur Übermittlung von Bildern aus der radiologischen Diagnostik sollten die Möglichkeiten der telemedizinischen Befundübermittlung verstärkt genutzt werden.

\section{Weiterverlegungskriterien}

Bei besonders schweren oder speziellen Verletzungen sollte nach Primärversorgung in einem regionalen Traumazentrum die zeitgerechte Weiterverlegung in ein ,überregionales“ Traumazentrum nach festgelegten Kriterien erfolgen (• Tab. 3). Hierbei sollte primär die Verlegung in das im lokalen Netzwerk zugeordnete überregionale Traumazentrum angestrebt werden.

Nach welchen Kriterien eine Weiterverlegung in ein überregionales Traumazentrum erfolgen soll, ist jeweils in Kooperation der Traumazentren in einem Netzwerk endgültig festzulegen.

Vor der Verlegung sollte die Transportfähigkeit des Patienten entsprechend der ATLS-Kriterien sichergestellt werden.

\section{Fazit für die Praxis}

\section{Numerisch gesehen verfügt die Bundes- republik Deutschland über ausreichend Krankenhäuser zur Behandlung Schwer- und Schwerstverletzter. Große Gebiete Deutschlands sind jedoch von einem op- timalen infrastrukturellen Versorgungs- profil weit entfernt. Eine adäquate und qualitätsorientierte Traumaversorgung kann aber nicht allein durch hierfür per- sonell und strukturell adäquat ausgerü- stete Krankenhäuser der Maximal- und Schwerpunktversorgung erreicht wer- den, sondern erfordert auch eine Anpas- sung der infrastrukturellen Bedingungen in den jeweiligen Bundesländern.}

\section{Korrespondierender Autor}

Prof. Dr. S. Ruchholtz

Klinik für Unfallchirurgie,

Universitätsklinikum Essen

Hufelandstraße 55, 45122 Essen

steffen.ruchholtz@uni-essen.de

Interessenkonflikt. Es besteht kein Interessenkonflikt. Der korrespondierende Autor versichert, dass keine Verbindungen mit einer Firma, deren Produkt in dem Artikel genannt ist, oder einer Firma, die ein Konkurrenzprodukt vertreibt, bestehen. Die Präsentation des Themas ist unabhängig und die Darstellung der Inhalte produktneutral.

\section{Literatur}

1. Albrink MH, Rodriguez E, England GJ et al. (1994) Importance of designated thoracic trauma surgeons in the management of traumatic aortic transection. South Med J 87: 497-501

2. Arbeitsgemeinschaft "Scoring" der Deutschen Gesellschaft für Unfallchirurgie (1994) Das Traumaregister der Deutschen Gesellschaft für Unfallchirurgie. Unfallchirurg 97: 230-237

3. Bauer H, Müller RT, Grusser C et al. (1986) Versorgungsstrategie beim schweren Schädel-Hirn-Trauma in der allgemeinchirurgischen Abteilung. Chirurg 57: 321-326

4. Biewener $A$, Aschenbrenner $U$, Rammelt $S$ et al. (2004) Impact of helicopter transport and hospital level on mortality of polytrauma patients. J Trauma 56: 94-98

5. Bouillon B, Kanz KG, Lackner CK et al. (2004) Die Bedeutung des Advanced Trauma Life Support ${ }^{\circ}$ $\left(\right.$ ATLS $\left.^{\circ}\right)$ im Schockraum. Unfallchirurg 107: 844-850

6. Cales RH, Ehrlich F, Sacra J et al. (1987) Trauma care system guidelines: improving quality through the systems approach. Ann Emerg Med 16: 464

7. Champion HR, Sacco WJ, Copes WS (1992) Improvement in outcome from trauma center care. Arch Surg 127: 333-338

8. Chan L, Bartfield JM, Reilly KM (1997) The significance of out-of-hospital hypotension in blunt trauma patients. Acad Emerg Med 4: 785-788

9. Compere V, Genevois A, Le Corre A et al. (2003) Influence of medical speciality and experience on interpretation of helicoidal thoracic computed tomography in blunt chest trauma. Intensive Care Med 29: 770-773

10. Culemann U, Seekamp A, Riedel U et al. (2003) Interdisziplinäres Polytraumamangement. Teil 2: Klinikaufnahme vital bedrohter traumatisierter Patienten. Notfall Rettungsmed 6: 573-579

11. Demarest GB, Scannell G, Sanchez K et al. (1999) In-house versus on-call attending trauma surgeons at comparable level I trauma centers: a prospective study. J Trauma 46: 535-542

12. Deo SD, Knottenbelt JD, Peden MM (1997) Evaluation of a small trauma team for major resuscitation. Injury 28: 633-637

13. Dodek P, Herrick R, Phang PT (2000) Initial management of trauma by a trauma team: effect on timeliness of care in a teaching hospital. Am J Med Q 15:3-8

14. Esposito TJ, Offner PJ, Jurkovich GJ et al. (1995) Do prehospital trauma center triage criteria identify major trauma victims? Arch Surg 1995: 171-176

15. Franklin GA, Boaz PW, Spain DA et al. (2000) Prehospital hypotension as a valid indicator of trauma team activation. J Trauma 48: 1034-1039
16. FriedI W, Karches C (1996) Das Schädel-Hirn-Trauma in der chirurgischen Akutversorgung. Primärversorgung in einem Krankenhaus der Grund- und Regelversorgung. Chirurg 67: 1107-1113

17. Fulda GJ, Tinkoff GH, Giberson F et al. (2002) Inhouse trauma surgeons do not decrease mortality in a level I trauma center. J Trauma 53: 494-502

18. Haas NP, Hoffmann RF, Mauch C et al. (1995) The management of polytraumatized patients in Germany. Clin Orthop 1995: 25-35

19. Haas NP, Fournier C von, A Tempka et al. (1997) Traumazentrum 2000. Wie viele und welche Traumazentren braucht Europa um das Jahr 2000? Unfallchirurg 100: 852-858

20. Hartmann J, Gabram S, Jacobs L et al. (1996) A model for an integrated emergency medicine/trauma service. Acad Emerg Med 3: 1136-1139

21. Havill JH, Sleigh J (1998) Management and outcomes of patients with brain trauma in a tertiary referral trauma hospital without neurosurgeons on site. Anaesth Intensive Care 26: 642-647

22. Helling TS, Nelson PW, Shook JW et al. (2003) The presence of in-house attending trauma surgeons does not improve management or outcome of critically injured patients. J Trauma 55: 20-25

23. Hulka F, Mullins RJ, Mann NC et al. (1997) Influence of a statewide trauma system on pediatric hospitalization and outcome. JTrauma 2: 514-519

24. Kane G, Wheeler NC, Cook S (1992) Impact of the Los Angeles County Trauma System on the survival of seriously injured patients. J Trauma 2: 576-583

25. Krötz M, Bode PJ, Häuser H et al. (2002) Interdisziplinäre Schockraumversorgung: Personelle, apparative und räumlich-logistische Konzepte in 3 Traumakrankenhäusern in Europa. Radiologe 42: 522-532

26. Kühne CA, Ruchholtz S, Sauerland S et al. (2004) Personelle und strukturelle Voraussetzungen der Schockraumbehandlung Polytraumatisierter - Eine systematische Literaturübersicht. Unfallchirurg 107: 851-861

27. Kühne CA, Ruchholtz S, Buschmann C et al. (in press) Initiative Traumanetzwerk DGU. Polytraumaversorgung in Deutschland - Eine Standortbestimmung. Unfallchirurg in press

28. Le Corre A, Genevois A, Benichou J et al. (1999) Interprétation des clichés thoraciques standard chez les traumatisés thoraciques: influence de l'expérience du lecteur. Ann Fr Anesth Reanim 18: 503-508

29. Le Corre A, Genevois A, Hellot MF et al. (2001) L'analyse du cliché thoracique chez le traumatisé n'est améliorée ni par une grille de lecture ni par l'expérience du lecteur. Ann Fr Anesth Reanim 20: 23-27

30. Leitlinien-Kommission der Deutschen Gesellschaft für Unfallchirurgie, Polytrauma (2001) Leitlinie für die unfallchirurgische Diagnostik und Therapie. Unfallchirurg 104: 902-912

31. Liener UC, Rapp U, Lampl L et al. (2004) Inzidenz schwerer Verletzungen - Ergebnisse einer populationsbezogenen Untersuchung. Unfallchirurg 107: 483-490

32. Lomas GA, Goodall O (1994) Trauma teams vs nontrauma teams. Accid Emerg Nurs 2: 205-210

33. Lossius HM, Langhelle A, Pillgram-Larsen J et al. (2000) Efficiency of activation of the trauma team in a Norwegian trauma referral centre. Eur J Surg 166: 760-764

34. Lucas CE, Dombi GW, Crilly RJ et al. (1997) Neurosurgical trauma call: use of a mathematical simulation program to define manpower needs. J Trauma 42: 818-824 
35. Mathiasen RA, Eby JB, Jarrahy R et al. (2001) A dedicated craniofacial trauma team improves efficiency and reduces cost. J Surg Res 97: 138-143

36. Mullins RJ, Mann NC (1999) Population-based research assessing the effectivness of trauma systems. J Trauma 47: 59-66

37. Norwood S, Myers MB (1994) Outcomes following injury in a predominantly rural-population-based trauma center. Arch Surg 29: 800-805

38. Palmer SH, Maheson M (1995) A radiological review of cervical spine injuries from an accident and emergency department: has the ATLS made a difference? J Accid Emerg Med 12: 189-190

39. Parzhuber A, Wiedemann E, Richter-Turtur M et al. (1994) Die Stellung des Allgemein- und Unfallchirurgen in der Neurotraumatologie: Erfahrungen und Ergebnisse aus 10 Jahren. Unfallchirurg 97: 615-618

40. Pohlenz O, Bode PJ (1996) The trauma emergency room: a concept for handling and imaging the polytrauma patient. Eur J Radiol 22: 2-6

41. Poon A, McCluskey PJ, Hill DA (1999) Eye injuries in patients with major trauma. J Trauma 46: 494-499

42. Ruchholtz S (2000) AG-Polytrauma. Das Traumaregister der Deutschen Gesellschaft für Unfallchirurgie als Grundlage des interklinischen Qualitätsmanagements in der Schwerverletztenversorgung. Unfallchirurg 103: 30-37

43. Ruchholtz S (2004) AG Polytrauma der DGU. Das externe Qualitätsmanagement in der klinischen Schwerverletztenversorgung. Unfallchirurg 107: 835-847

44. Ruchholtz S, Nast-Kolb D, Waydhas C et al. (1994) Frühletalität bei Polytrauma. Eine kritische Analyse vermeidbarer Fehler. Unfallchirurg 7: 285-291

45. Ruchholtz S, Waydhas C, Lewan U et al. (2002) A multidisciplinary quality management system for the early treatment of severely injured patients: implementation and results in two trauma centers. Intensive Care Med 28: 1395-1404

46. Rutledge R, Fakhry SM, Meyer A et al. (1993) An analysis of the association of trauma centers with per capita hospitalizations and death rates from injury. Ann Surg 18: 512-521

47. Sava J, Alo K, Velmahos GC et al. (2002) All patients with truncal gunshot wounds deserve trauma team activation. J Trauma 52: 276-279

48. Shackford SR, Hollingworth-Fridlund P, Cooper GF et al. (1986) The effect of regionalization upon the quality of trauma care as assessed by concurrent audit before and after institution of a trauma system: a preliminary report. J Trauma 26: 812-820

49. Shackford SR, Hollingsworth-Fridlund P, McArdle $M$ et al. (1987) Assuring quality in a trauma system - the Medical Audit Committee: composition, cost and results. J Trauma 27: 866-875

50. Shackford SR, Mackersie RC, Hoyt DB et al. (1987) Impact of a trauma system on outcome of severely injured patients. Arch Surg 122: 523-527

51. Sturm JA, Lackner CK, Bouillon B (2002) Advanced Trauma Life Support (ATLS). Unfallchirurg 105: 1027-1032

52. Tinkoff GH, O'Connor RE (2002) Validation of new trauma triage rules for trauma attending response to the emergency department. J Trauma 52: 1153 1159

53. Velmahos GC, Fili C, Vassiliu P et al. (2001) Aroundthe-clock attending radiology coverage is essential to avoid mistakes in the care of trauma patients. Am Surg 67: 1175-1177

54. Vorhies RW, Harrison PB, Smith RS et al. (2002) Senior surgical residents can accurately interpret trauma radiographs. Am Surg 68: 221-226
55. Wachtel TL, Coniglio R, Bourg P et al. (2001) The synergistic relationship between a level I trauma center and a regional pediatric trauma center. Semin Pediatr Surg 10: 38-43

56. Wenneker WW, Murray DH Jr, Ledwich T (1990) Improved trauma care in a rural hospital after establishing a level II trauma center. Am J Surg 160: 655658

57. Wissenschaftlicher Arbeitskreis Neuroanästhesie der Deutschen Gesellschaft für Anästhesiologie und Intensivmedizin, Arbeitsgemeinschaft Intensivmedizin und Neurotraumatologie der Deutschen Gesellschaft für Neurochirurgie, Sektion Rettungswesen und Katastrophenmedizin der Deutschen Interdisziplinären Vereinigung für Intensivund Notfallmedizin (2000) Empfehlungen zur Erstversorgung des Patienten mit Schädel-Hirn-Trauma bei Mehrfachverletzung. In: Opderbecke HW, Weißbauer W (Hrsg) Entschließungen - Empfehlungen -Vereinbarungen - Ein Beitrag zur Qualitätssicherung in der Anästhesiologie. Deutsche Gesellschaft für Anästhesiologie und Intensivmedizin e. V. (DGAI), Nürnberg,http://www.dgai.de/ 06pdf/5_13.pdf

58. Zulick LC, Dietz PA, Brooks K (1991) Trauma experience of a rural hospital. Arch Surg 26: 14 